\title{
Ultrafast and long-range self-transport of droplets on multi-bioinspired surface with periodic gradient structure
}

\section{Dongdong Xie}

Shanghai Jiao Tong University https://orcid.org/0000-0003-1717-099X

\section{Guilian Wang}

Shanghai University of Engineering Science

\section{Yunna Sun}

Shanghai Jiao Tong University

\section{Chaofeng Wu}

Shanghai Jiao Tong University

Guifu Ding ( $\sim$ gfding@sjtu.edu.cn )

Shanghai Jiao Tong University

\section{Article}

Keywords: Heterogeneous Wettability, Shape Gradient Morphology, Droplet Manipulation Strategy

Posted Date: July 13th, 2021

DOI: https://doi.org/10.21203/rs.3.rs-668754/v1

License: (c) (1) This work is licensed under a Creative Commons Attribution 4.0 International License. Read Full License 


\title{
Ultrafast and long-range self-transport of droplets on multi-bioinspired surface
}

\section{with periodic gradient structure}

\begin{abstract}
Droplet self-transport is crucial in various fields ranging from physics to biochemistry. Despite extensive progress, existing systems for droplet self-transport still perform at low transport velocity or limited transport range. Here, a multi-bioinspired surface comprising two-dimensional gradient structures is proposed innovatively, which integrates the heterogeneous wettability with the shape gradient morphology. Droplet transport behaviors are systematically investigated from experiment, theory, and simulation. The fabricated structure achieves the ultrafast (over $\sim 450 \mathrm{~mm} / \mathrm{s}$ ) and longrange (over $\sim 200 \mathrm{~mm}$ ) self-transport of droplets. Moreover, the fantastic scalability of this structure enables it to pump numerous multi-scale droplets from one site to the preset region with ultralow loss. Drawing inspirations, two systems have been designed to complete complex tasks on open surface. This work provides a reliable droplet manipulation strategy for various applications, such as water collection, microfluidics, and biomedicine, etc.
\end{abstract}

\section{Introduction}

The self-transport of droplets along a particular direction plays an indispensable role in various fields involving but not limited to microfluidics ${ }^{1-3}$, liquid collection/separation ${ }^{4-6}$, antifogging ${ }^{7-9}$, and biomedical equipment ${ }^{10-12}$. Millions of years of natural evolution have always provided us with fantastic inspiration for manipulating droplets ${ }^{13}$. The back of the desert beetle is a heterogeneous wettability surface, where the hydrophilic patterns are surrounded by hydrophobic background, effectively facilitating water collection for surviving ${ }^{14}$. The cactus spines possess an asymmetric topological structure with a gradient of curvature, which incurs an unbalanced Laplace pressure acting on droplet, thus propelling the droplet to move from the tip to the $\operatorname{root}^{15}$. The periodic microcavities are regularly distributed on the peristome surface of Nepenthes alata, which contributes to continuous long-range droplet self-transport ${ }^{16}$. Some other organisms have also evolved similar functions ${ }^{17-20}$. These amazing natural phenomena are mainly attributed to the wettability gradient or anisotropic structure, which produces the unbalanced surface tension to drive droplets to diffuse or transport along a particular direction ${ }^{21-25}$. Drawing inspirations, amounts of research has been devoted to the development of functional surfaces with wettability gradient or asymmetric structure to achieve rational manipulation of droplets ${ }^{26-29}$. Despite extensive progress, the self-transport of droplets based on existing strategies is still restricted by low transport velocity and limited transport range ${ }^{30-32}$. Additionally, previous studies have shown that complex 3D micronano structures are often indispensable for droplet self-transport ${ }^{33-37}$. This not only greatly increases the complexity of manufacturing, but also reduces its compatibility with other systems, which may greatly limit their further development as well as practical applications. Noteworthily, it is of great challenge to losslessly transport considerable droplets from one site to another since amounts of liquid will remain on the track during the transport process ${ }^{24,28,34,38}$. 
Here in, we for the first time propose a multi-bioinspired surface comprising two dimensional gradient structures, which integrates the heterogeneous wettability with the anisotropic structure. To deeply understand of the proposed structure, the droplet transport behaviors have been systematically investigated from experiment, theory, and simulation, which are in good agreement. In addition to the ultrafast self-transport, e.g., $\sim 451.20 \pm 6.68 \mathrm{~mm} / \mathrm{s}(\sim 305.05 \pm 8.02 \mathrm{~mm} / \mathrm{s})$ on the wet (dry) track in 15-mm displacement, the fabricated surface achieves the long-range self-transport of droplets (over $\sim 200 \mathrm{~mm}$ ). Besides, the novel structure can pump considerable droplets from one site to the preset region with ultralow loss, which is similar to South-to-North Water Diversion ${ }^{39}$, but this strategy does not require external energy. Moreover, the proposed structure is scaleable. Coupled with the advanced manufacturing technology, it is even expected to rationally manipulate droplets at the micro/nano scale. Drawing inspirations, we further design two systems to complete complex tasks on open surface. In the broad application prospects, this work will open a new avenue for the wide application ranging from fluid to energy.

\section{Results}

\subsection{Design principles}

The designed principles of the proposed surface (Fig. 1) are mainly originated from three distinct bioinspirations, namely the Janus wettability of desert beetle, the tapered asymmetric of cacti spine, and the periodic characteristic structure of Nepenthes alata. The difference of surface energy leads to the formation of "potential well" namely superhydrophilic area on the open surface, the boundary of which can effectively pin the liquid contact line. By adjusting the shape of the "potential well", the unbalanced surface tension can be produced, which actuates the spontaneous droplet transport within the superhydrophilic track. The periodic structure can be effectively combined, contributing to continuous long-range transport of droplets. Moreover, it makes the droplets flowing into the track at different spatial positions (the same position of different unit) possess a similar dynamic property, such as approximate initial velocity, etc.

\subsection{Droplet transport behaviors}

Fig. 2a shows the scanning electron microscopy (SEM) images of the multi-bioinspired surface, exhibiting that the interconnected architectures of $\mathrm{Cu}(\mathrm{OH})_{2}$ nanowires completely cover the substrate. $\mathrm{Cu}(\mathrm{OH})_{2}$ was chosen to be the basic composition of Janus surface due to its intrinsic superhydrophilic and simple fabrication. The detailed manufacturing of the multi-bioinspired surface and its structural parameters can be found in Supplementary Fig. 1. The advanced manufacturing technology can precisely fabricate the Janus structure with various complex patterns even at the micro-nano scale (Supplementary Fig. 2). Moreover, the technical route is suitable for almost any substrate, such as glass, metal, ceramics, polydimethylsiloxane (PDMS) and so on, which greatly enhances its application potential. The water contact angle of the superhydrophobic and superhydrophilic area are $155 \pm 1.5^{\circ}$ and $\sim 0^{\circ}$, respectively (See Supplementary Fig. 3 for the rapid droplet spreading on a superhydrophilic $\mathrm{Cu}(\mathrm{OH})_{2}$ surface). Noteworthily, the morphology of Janus surface shows little difference between superhydrophilic pattern and superhydrophobic background. Therefore, the change in the surface chemical composition is responsible for the 
formation of the wettability difference, which is further confirmed by the energy-dispersive spectroscopy (EDS) results (Fig. 2b).

The behaviors of droplets on a fabricated surface were caught by high-speed camera. A $7 \mu \mathrm{L}$ droplet self-transport was achieved on the dry superhydrophilic track (Fig. 2c and Supplementary Movie 1). The droplet was placed upon the narrow end of the first unit, and the precision displacement device was controlled to make the droplet approach the Janus surface very slowly (the input energy is negligible, Fig. 2c (i)). Once in contact with the superhydrophilic track, the droplet was instantly captured and immediately detached from the syringe needle in $\sim 8 \mathrm{~ms}$. Meanwhile, an advancing liquid front rapidly propagated ahead of the bulk of liquid (liquid bulge) towards the wide end of the current unit, and then continued to enter the next unit. The liquid bulge exhibited a relatively sluggish start owing to inertia, but it sped up subsequently to keep up with the liquid front (Fig. 2c (ii-iii)). Upon arrival at the end of the unit, a part of the liquid flowed into the next unit maintaining the original motion, while the remaining liquid flowed back to the narrow end of the current unit (Fig. 2c (iv)). The returned liquid damply oscillated back and forth in the unit. During each oscillation, a part of liquid would flow into the forward units. Within $\sim 200 \mathrm{~ms}$, the motion of liquid bulge in the current unit reached quasi-static state which is defined as the apparent velocity of horizontal motion approaching 0 (Fig. 2c (v)). In quasi-static state, the remaining liquid bulge would stay at the broad area of the unit acting as a "source" that replenishes fluid for movement ahead. As shown in Fig. 2c (v), the advancing liquid front transported $\sim 19.43 \mathrm{~mm}$ forward in 200 ms. Finally, a $7 \mu \mathrm{L}$ droplet spread over the entire superhydrophilic track in $\sim 8 \mathrm{~s}$ (the total area is $\sim 135.30 \mathrm{~mm}^{2}$, Fig. 2c (vi)).

Fig. $2 d$ shows the self-transport behaviors of numerous droplets $(\sim 200 \mu \mathrm{L})$ on the wet track. A thin water film was automatically formed on the superhydrophilic track after the first droplet transported (Fig. 2d (i)), and subsequent droplets exhibited similar transport behaviors, but the velocity would increase obviously (Fig. 2d (i-iv), see Supplementary Movie 1 for details). With the gradual release of droplets, the liquid level of each unit rose, and the unit farther from the depositing site gave a smaller rise (Fig. $2 \mathrm{~d}(\mathrm{v})$ ). Once the release stopped, the liquid level in each unit would decline rapidly while the liquid level in the preset region continued to rise, showing that considerable droplets continuously transported towards the preset region. When the system reached equilibrium, only a thin water film was retained on the track while most of the liquid was transported to the preset region, namely reservoir (Fig. 2d (vi), see Supplementary movie 2 for the whole process). Noteworthily, when the depositing site is in the middle of track, the released droplets can still be directionally pumped to the preset region eventually (Supplementary Fig.4 and Movie 2).

To further demonstrate the advantages of proposed structure in long-range droplet transport, 20 units were compounded to form a track over $\sim 200 \mathrm{~mm}$. Numerous droplets $(\sim 900 \mu \mathrm{L})$ were continuously pumped to the preset region, forming a growing bulge there and leaving a thin water film on the track (Fig. 2e, see Supplementary Fig. 5 and Movie 3 for details).

\subsection{The underlying mechanism of droplet transport behaviors}

\section{Force analysis of liquid bulge}


The underlying mechanism of droplet transport on the fabricated multi-bioinspired surface has been systematically investigated (Fig. 3). The initial propagation of the advancing liquid front on the dry superhydrophilic track is driven by hemiwicking through the micro-/nanoscale roughness features ${ }^{40}$ derived from $\mathrm{Cu}(\mathrm{OH})_{2}$ nanowire. Nevertheless, the motion of the liquid bulge is strongly affected by the Laplace force $\left(F_{\mathrm{L}}\right)$ arising from the shape gradient, the pinning force $\left(F_{\mathrm{P}}\right)$ derived from the Janus structure, the hysteresis resistance force $\left(F_{\mathrm{H}}\right)$ and the hydrodynamic resistance force $\left(F_{\mathrm{D}}\right)$ formed by the track surface. On the fabricated surface, the resultant force on the liquid bulge along the advancing direction is

$$
F=F_{\mathrm{L}}-F_{\mathrm{P}}-F_{\mathrm{H}}-F_{\mathrm{D}}
$$

Along the superhydrophilic track, the contact angle differential between droplet front $\left(\theta_{\mathrm{f}}\right)$ and back $\left(\theta_{b}\right)$ will engender unbalanced surface tension, namely Laplace force which can be expressed as follows ${ }^{41}$ :

$$
F_{\mathrm{L}}=\gamma_{L G} l_{T C L}\left(\cos \theta_{\mathrm{f}}-\cos \theta_{\mathrm{b}}\right)
$$

where $l_{T C L}$ denotes the length of three phase contact line, and $\gamma_{L G}$ is the water surface tension $(\sim 72.8 \mathrm{mN} / \mathrm{m}$ at room temperature). At the edge of the Janus structure, there exists an energy barrier that pinned the liquid contact line. The apparent contact angle along the edge $\left(\theta_{\mathrm{e}}\right)$ is controlled by the liquid volume and the local track width ${ }^{42}$, namely the confinement effect (Fig. 3a (i-iii)). Noteworthily, $\theta_{\mathrm{e}}$ varies along the transport length $x$, which satisfies $\theta_{s i} \leq \theta_{\mathrm{e}} \leq \theta_{s o}$, where $\theta_{s i}$ and $\theta_{s o}$ are the apparent contact angle on the superhydrophilic and superhydrophobic surface without confinement, respectively. Then, the pining force arising from the Janus structure edge can be obtained ${ }^{43}$

$$
F_{\mathrm{P}}=\gamma_{L G} l_{T C L} \cos \overline{\theta_{\mathrm{e}}} \sin \varphi
$$

where $\varphi$ is the half apex angle of the wedge-shape. The hysteresis resistance force impedes the droplet movement which can be expressed as ${ }^{27}$

$$
F_{\mathrm{H}}=\gamma_{L G} l_{T C L}\left(\cos \theta_{\mathrm{r}}-\cos \theta_{\mathrm{a}}\right)
$$

where $\theta_{\mathrm{r}}$ and $\theta_{\mathrm{a}}$ represent the receding and advancing contact angle, respectively. Noteworthily, the droplet would rapidly spread on the superhydrophilic track of the fabricated Janus surface, indicating that both of $\theta_{\mathrm{r}}$ and $\theta_{\mathrm{a}}$ approximated $\sim 0^{\circ}$. Therefore, the effect of $F_{\mathrm{H}}$ (approaching $\sim$ ) on the droplet movement was negligible in this work. In addition to $F_{\mathrm{H}}$, there exists the hydrodynamic resistance force generated by the delivery substrate, which inclines to resist the movement of droplet. The approximated $F_{\mathrm{D}}$ can be expressed as ${ }^{44}$

$$
F_{\mathrm{D}} \approx \frac{6 \pi R(t) \mu U}{\theta_{\mathrm{avg}}(t)} \ln (2 \epsilon)
$$

where $\mu, U, R(t)$ are the dynamic viscosity, velocity, and equivalent wet radius of the droplet, respectively. $\theta_{\text {avg }}(t) \approx\left(\theta_{\mathrm{f}}(t)+\theta_{\mathrm{b}}(t)\right) / 2$, which is the average contact angle of the droplet. The dimensionless parameter $\epsilon=l_{s} / R(t)$ where $l_{s}$ denotes the slip length of the droplet.

When the droplet is deposited at the unit narrow end, $F_{\mathrm{L}}$ is the driving force since $\theta_{\mathrm{b}}$ is larger than $\theta_{\mathrm{f}}$ (Fig. 3a (iv)). Besides, $F_{\mathrm{P}}$ works as the driving force in the early stage of droplet transport where $\overline{\theta_{\mathrm{e}}}>90^{\circ}$ since $\theta_{\mathrm{e}}>90^{\circ}$ for most sections of the liquid bulge owing to the confinement 
effect (Fig. 3a (i)). Under the assistance of the driving force $\left(F_{\mathrm{L}}\right.$ and $\left.F_{\mathrm{P}}\right)$, the droplet could overcome the resistance force $\left(F_{\mathrm{D}}\right)$ to transport from the narrow end to the wide end. Upon arrival at the far downstream portion of the unit, $\overline{\theta_{\mathrm{e}}}$ becomes less than $90^{\circ}$ (Fig. 3a (ii)), thus $F_{\mathrm{P}}$ turns to be the resistance force. Moreover, the track width at the end of the unit decreases, which blocks a portion of the liquid bulge from flowing forward to the next unit, causing $\theta_{\mathrm{f}}$ to rise. Coupled with the narrowed track there, $\theta_{\mathrm{f}}$ becomes larger than $\theta_{\mathrm{b}}$, which makes $F_{\mathrm{L}}$ the resistance force (Fig. 3a (v)). Therefore, a part of the liquid flows back under the combined effect of $F_{\mathrm{P}}$ and $F_{\mathrm{L}}$. Eventually, the resistance of $F_{\mathrm{D}}$ to the droplet motion is gradually enhanced with the increasing wet area (Equation (5)), forcing droplet transport to cease.

After the thin water film is automatically formed, the transport behaviors of subsequent droplets are similar to that before, but these droplets move obviously faster. This can be explained that the thin water film blocks the sequential liquid from directly contacting with the track surface, resulting in the drastic reduction of $F_{\mathrm{D}}$. Similar phenomena were also reported in previous studies $^{45,46}$. Drawing inspiration, we investigated droplet transport on a fabricated surface comprising hydrophilic-superhydrophobic Janus structure. The dry hydrophilic track cannot achieve continuous droplet transport owing to the non-ignorable $F_{\mathrm{H}}$ (Supplementary Fig. 6a). Nevertheless, after artificial pre-wetting, numerous droplets $(\sim 80 \mu \mathrm{L})$ can successfully transport to the preset region through the wet hydrophilic track due to the sharply reduction of $F_{\mathrm{D}}$ as well as $F_{\mathrm{H}}$ (Supplementary Fig. 6b and Movie 4). This interesting discovery expands the application scenarios and environmental adaptability of the proposed structure to a certain extent.

\section{Analysis from the perspective of system energy}

To promote a deeper understanding of droplet transport behaviors on the fabricated multibioinspired surface, a careful analysis was performed from the perspective of system energy. Herein, $E_{0}$ represents the droplet surface energy in the initial state (Fig. 3b). In this case, the droplet is infinitely close to the superhydrophilic track. For a given volume of droplet, $E_{0}$ is a constant. $E_{i}$ denotes the surface energy of droplet located on the Janus surface, which changes with the droplet transport. Define the initial state of the droplet is the zero potential energy state $\left(E_{0}=0\right)$, thus the surface energy of a droplet $\left(E_{i}\right)$ can be expressed as follows:

$$
E_{i}=E_{i}-E_{0}=\left(A_{i}-A_{0}\right) \gamma_{L G}+\left(B_{i}-B_{0}\right) \gamma_{S G}+\left(C_{i}-C_{0}\right) \gamma_{S L}
$$

where $\gamma$ is the interfacial tension between the gas $(G)$, liquid $(L)$ and solid $(S) ; A, B$ and $\mathrm{C}$ denote the corresponding contact areas. During the droplet transport, the relation between $E_{i}$ and $\theta_{i}$ (transient water contact angle) can be obtained that

$$
E_{i}=\lambda\left(\cos \theta_{i}^{3}-3 \cos \theta_{i}+2\right)^{\frac{1}{3}}-\eta
$$

where $\lambda=\gamma_{L G}\left(9 \pi V_{i}^{2}\right)^{\frac{1}{3}}, \quad \eta=A_{0} \gamma_{L G}$ and $V_{i}$ denotes the volume of the droplet (See Supplementary Note 1 for the full mathematic derivation). Obviously, $\lambda$ and $\eta$ can be treated as constants at a given volume of droplet. Thus, $E_{i}{ }^{\prime}\left(\theta_{i}\right)=3 \lambda \sin ^{3} \theta_{i}$. For $\theta_{i} \in\left[0^{\circ}, 180^{\circ}\right], E_{i}{ }^{\prime}\left(\theta_{i}\right) \geq$ 0 . Therefore, the surface energy of the droplet can be precisely reflected by the contact angle. For a 
droplet at a certain position on the track, the larger $\theta_{i}$ corresponds to the greater $E_{i}$, indicating the smaller released energy $\left(\Delta E_{s}\right)$ during transport process. If $\theta_{i}$ approaches to $\sim 0^{\circ}$ (superhydrophilic surface), $E_{i}$ is approximately equal to $-\eta$. Thus $\Delta E_{s}$ can be obtained

$$
\Delta E_{S} \approx 4 \pi R_{0}^{2} \gamma_{L G}
$$

where $R_{0}$ is the equivalent radius of the droplet in the initial state. Obviously, a droplet with a larger volume gives a greater surface energy. Considering the influence of gravity, $R_{0}$ was modified properly in combination with the theoretical calculation and experimental observation (See Supplementary Note 2).

Under the premise of sufficient driving energy, the droplet has a propensity to spread over the entire dry superhydrophilic track, as that leads to declining of $\theta_{i}$, resulting in a lower surface energy (Fig. 3b (i)). Specifically, in an asymmetric unit of the fabricated surface, $\theta_{i}$ of droplet at the narrow end is larger than that at the wide end due to the confinement effect, then it can be obtained that $E_{1}>E_{2}$ (Equation (7)). Therefore, the droplet tends to transport toward wide end in the current unit. Besides, the liquid bulge inclines to enter the forward dry unit instead of flowing back to the wet narrow end, as that causes declining of $\theta_{i}$, namely $E_{2}>E_{3}$. With the increase of wet area, the resistance work increases. Until the driving energy is consumed, the droplet transport ceases.

Once the water film is formed, the energy consumed by $F_{D}$ will dramatically decline. In other words, the transport of subsequent droplets is almost lossless on the track. Thus, droplets could transport to any position of the wet track (reservoir included). For each unit, the increased surface energy $\left(\Delta E_{S i}, i=1-n\right)$ caused by the introduction of a droplet $\left(V_{0}\right)$ is theoretically equal due to the periodicity, namely $\Delta E_{S 1}=\Delta E_{S 2}=\cdots=\Delta E_{S n}$ ((Fig. 3b (ii)). Nevertheless, the preset region for droplets collection is relatively unrestricted compared with the unit, so the increase of $\theta_{i}$ there caused by the same volume is smaller, leading to the lower increased surface energy there $\left(\Delta E_{S R}\right)$, namely $\Delta E_{S 1}=\Delta E_{S 2}=\cdots=\Delta E_{S n}>\Delta E_{S R}$. Therefore, in order to minimize the energy of the whole system, no matter where the droplet is deposited on the wet track, it will eventually transport to the preset region.

As the droplets continue to release, the wide end of the unit as well as reservoir will accumulate liquid bulges of different size (Fig. 3b (iii)). After the release stops, the liquid level in the unit decreases in varying degrees, thus $\theta_{i}(i=1-n)$ declines, resulting in the decrease of $E_{i}$. The corresponding variable is just the opposite in the reservoir. Noteworthily, the reduced surface energy ( $\left.\Delta E_{i}, i=1-\mathrm{n}\right)$ on the superhydrophilic track is larger than that increased in the reservoir $\left(\Delta E_{R}\right)$, namely $\Delta E_{1}+\Delta E_{2}+\cdots+\Delta E_{n}>\Delta E_{R}$. Therefore, the track continuously pumps droplets towards the preset region and accumulate it in the form of growing bulge.

During the droplet transport process on the dry track, the driving energy $\left(\Delta E_{S}\right.$ and $\left.\Delta E_{G}\right)$ is mainly used to overcome the hydrodynamic work. Respect to the wet track, the driving energy of droplets is almost converted to the surface energy and gravitational potential energy of the liquid bulge in reservoir.

\subsection{Droplets transport performance and dynamic characteristics}


According to section 2.3, the Janus wettability and anisotropic structure effectively incur the confinement effect, causing the anisotropy of the apparent contact angle and asymmetry of the contact line. Therefore, the intensity of the confinement effect has a crucial influence on droplet transport. For the proposed structure, the gap width (the narrowest site, see Supplementary Fig. 1b) not only directly affects the intensity of confinement effect, but also plays an important role in the continuous transport of droplets between adjacent units. Hence, it is of great significance to investigate the critical design parameter, namely gap width.

Supplementary Table 1 lists the structural parameters of each sample in detail. The gap width of the track varies from $500 \mu \mathrm{m}$ to $1000 \mu \mathrm{m}$, and the interval is $100 \mu \mathrm{m}$. A droplet $(5 \mu \mathrm{L})$ was deposited from the narrow end of a unit. In the early stage, the backward contact angle in experimental observation $\left(\theta_{b e}\right.$ ) decreases from $\sim 45^{\circ}$ to $\sim 22^{\circ}$ with the increase of the gap width from $500 \mu \mathrm{m}$ to $1000 \mu \mathrm{m}$ (Fig. 4a (i)), which is in good agreement with the simulation results (Fig. 4a (ii)). This can be explained that the narrower position gives the stronger confinement effect. Noteworthily, the smaller gap width gives the longer track of the unit at the given apex angle $\left(10^{\circ}\right)$ and maximum width $(2 \mathrm{~mm})$. Therefore, track with smaller gap width gives the continuous stronger confinement effect. As a result, the narrower gap always exhibits the larger $\theta_{b}$, corresponding to the greater $F_{L}$ when $\theta_{f}$ is almost equal. Additionally, the extent of liquid axial expansion at the pinning edge decreases with the increase of the gap (Fig. 4a (iii)). The larger expansion extent gives the greater pinning force along the edge. Therefore, the narrower track gives the larger driving force acting on the liquid bulge in the early stage, which is manifested by the larger pressure difference along the flow direction (Fig. 4a (iii)).

Nonetheless, the narrower gap is not conducive to the continuous transport of droplets between the units due to its larger potential barrier. Here, the potential barrier is characterized by the following indexes, namely the volume and the transport range of liquid flowing into the adjacent unit, and the extent of liquid bulge blocked at the gap. Obviously, the narrower gap gives the less liquid and the shorter transport range as well as the larger liquid bulge (Fig. 4b (i)), which is consistent with the simulation results (Fig. 4b (ii, iii)). This may be explained that the droplet surface will deform when it flows through the gap, resulting in the increase of its surface energy. The smaller gap gives the larger deformation, corresponding to the larger potential barrier, which is manifested by the larger relative pressure at the gap (Fig. $4 \mathrm{~b}$ (iii)).

Based on the above analysis, it is speculated that there exists an optimal gap width to make the droplet exhibit the best transport performance. A droplet $(5 \mu \mathrm{L})$ was released at the narrow end of the first unit on the wet track. The recovery time of liquid level in the first unit is used to evaluate the droplet transport performance, and the shorter recovery time gives the better performance. The results showed that the recovery time was less than $10 \mathrm{~s}$ for gap widths of $1000 \mu \mathrm{m}$ and $900 \mu \mathrm{m}$, less than $30 \mathrm{~s}$ for gap width of $800 \mu \mathrm{m}$, more than $50 \mathrm{~s}$ for gap widths of $700 \mu \mathrm{m}$ and $600 \mu \mathrm{m}$, and the continuous transport of droplet cannot be realized for gap width of $500 \mu \mathrm{m}$. Considering the effect of gap width on the driving force and the potential barrier, the proposed structure with 900 $\mu \mathrm{m}$ gap width gives the optimal transport performance in this work. Hence, the dynamic 
characteristics of droplets transport on the track with $900 \mu \mathrm{m}$ gap width are systematically investigated (Fig. 4c-f).

Each plot in Fig. 4c-f represents the average reading of at least five independent runs, with the error bars showing the standard deviation. Fig. $4 \mathrm{c}$ shows the droplet average velocity with respect to displacement and volumes on the dry superhydrophilic track. Intuitively, the average velocity of droplet successively decreases with the increase of displacement, but the descent rate varies with volume (Supplementary Fig. 7). Over the initial length of $9 \mathrm{~mm}$, the average velocity of different droplet volumes is very close. In the next $6 \mathrm{~mm}$ displacement, the average velocity of the $3 \mu \mathrm{L}$ droplet rapidly decrease, which is significantly lower than that of $5 \mu \mathrm{L}$ and $7 \mu \mathrm{L}$ droplet. A sharp drop in average velocity occurs in the last $3 \mathrm{~mm}$ for $5 \mu \mathrm{L}$ droplet. In the studied displacement range, the average velocity of $7 \mu \mathrm{L}$ droplet decreased steadily. Under the same conditions (volume and displacement), the average velocities of advancing liquid front on the wet track are greater than that on the dry track (Fig. 4d). This is attributed to the sharply reduction of $F_{D}$. Noteworthily, as the transport displacement increases, the corresponding average velocities with different volume droplets merely slightly decreases, floating around $446.50 \mathrm{~mm} / \mathrm{s}$. Considering the effect of $F_{D}$, the dynamic characteristics of droplet on the dry track are further analyzed.

The velocity-time and displacement-time (on $x-t^{1 / 2}$ axes) profiles of the advancing liquid front on the dry track are depicted in Fig. 4e and Fig. 4f, respectively. For a given volume of droplet, the transport velocity first decreases oscillatorily, then increases sharply, and finally decreases with time. Taking $5 \mu \mathrm{L}$ droplet as an example, the velocity decreases oscillatorily from $343.53 \pm 7.72 \mathrm{~mm} / \mathrm{s}$ to $200.71 \mathrm{~mm} / \mathrm{s}$ in $\sim 11 \mathrm{~ms}$. The large initial velocity is attributed to the release of large surface energy. The advancing liquid front transport forward which is akin to liquid hemiwicking, and the velocity decays under the action of $F_{D}$. Therefore, it exhibits the Washburn profiles ${ }^{47}$ for the displacementtime which shows the linear relation on the $x-t^{1 / 2}$ (before $16 \mathrm{~ms}$ in Fig. 4f). The liquid bulge shows a relatively hysteresis owing to its inertia, but it quickly keeps up with the advancing liquid front under the action of $F_{p}$ and $F_{L}$. This results in a dramatic variation in velocity which dramatically rises to $416.86 \pm 17.83 \mathrm{~mm} / \mathrm{s}$ within $\sim 4 \mathrm{~ms}$ and then decrease to $258.61 \pm 14.78 \mathrm{~mm} / \mathrm{s}$ in $\sim 9 \mathrm{~ms}$. Thereafter, the liquid bulge acts as a "source" that provides the driving potential for the advancing liquid front to overcome the resistance of $F_{D}$ to propagate further forward. Therefore, the droplet transport velocity floats around $261.61 \mathrm{~mm} / \mathrm{s}$ within $\sim 9 \mathrm{~ms}$, the stage of which is defined as the stationary period of droplet transport. After the liquid bulge is almost consumed, the transport velocity continuously decreases to $96.50 \pm 7.72 \mathrm{~mm} / \mathrm{s}$ in $19 \mathrm{~ms}$.

As for different volumes of droplet, the variation trend of velocity with transport time is basically similar, but the stationary period is different. The increase of droplet volume prolongs the stationary period. The stationary period of $3 \mu \mathrm{L}$ droplet is close to $0 \mathrm{~ms}$, that of $5 \mu \mathrm{L}$ droplet is about $9 \mathrm{~ms}$, and that of $7 \mu \mathrm{L}$ droplet increases to $\sim 21 \mathrm{~ms}$. This can be explained that the larger droplet volume gives the greater surface energy, making the liquid bulge a larger driving potential, thus prolonging the stationary period as well as total transport range (Fig. 4f). Similarly, the variation of average velocity with transport displacement in Fig. $4 \mathrm{c}$ is also explained reasonably. Based on this, 
we can extrapolate that the average transport velocity of $7 \mu \mathrm{L}$ droplet will also decline sharply with the further increase of the transport displacement. Noteworthily, the dynamic characteristics of droplet with different volumes are basically the same before the stationary period, e.g., the average velocities in 9-mm displacement (Fig. 4c), the instantaneous velocity (Fig. 4e) and displacement (Fig. 4f) before $\sim 25 \mathrm{~ms}$. Until the liquid bulge is needed to provide driving potential for the further forward propagation of the advancing liquid front, the dynamic characteristics of droplet with different volumes begin to apparently differentiate.

\subsection{Completing complex tasks on open surface}

To explore the application potential of the proposed structure, we further prove its scalability (Supplementary Fig. 8a, Movie 5). The fantastic scalability of the structure enables it to rationally manipulate a wide range of liquid volumes. Drawing inspirations, we designed two systems based on the bioinspired structure to complete complex tasks on open surface.

Both designed systems consist of two kinds of proposed structure with different scales (Track I \& II, see Supplementary Fig. 8b,c for details). Tracks I are placed parallel to each other, and a rectangular superhydrophilic strip is protruded from the first unit of track II to facilitate the droplet draining process. The difference between the two systems mainly lies in the number of Track II, which makes their functions and potential application scenarios quite different. The first system integrates metering, merging and ultrafast long-range transport of droplets (Fig. 5a). On this basis, the second system added the function of droplet splitting (Fig. 5b). To understand the whole process more clearly, a series of close-up snapshots of the droplet merging are given (Fig. 5c, see Supplementary Movie 11 for details), which are basically the same between the two systems.

As for the first system, droplets were continuously released at the narrow end of the first unit (track I), and then rapidly transported to the preset region, forming a growing bulge there (Fig. 5a (i-ii)). After each track transported droplets of $\sim 23 \mu \mathrm{L}$, the two liquid bulges contacted each other at the largest girth (Fig. 5a (iii)). Then the two bulges merged to form a liquid bridge which grew rapidly. The liquid bridge kept axial expansion owing to the inertial effect, obtaining an oblong shape of the merged bulge. When the merged bulge touched the rectangular strip, it expanded along the height and lateral direction at the expense of axial contraction (Fig. 5a (iv-v)). Sharp fluctuations in the shape of merged bulge can be observed since the merging of two volumes released great surface energy. Subsequently, the merged bulge continuously advanced onto track II through the rectangular strip, and then its de-bridging over the strip was observed at $t=160 \mathrm{~ms}$, leaving two small bulges on track I (Fig. 5a (vi)). Finally, the bulge on track II rapidly transported to the preset region (Fig. 5a (vii)). Noteworthily, the process of droplets metering, merging and transport is repeatable. After eight cycles (Supplementary Movie 6), the system successfully manipulated considerable droplets of $\sim 368 \mu \mathrm{L}$, and most of the droplets were pumped to the preset region of track II (Fig. 5a (viii)). This system is expected to utilized in water collection and drug delivery, etc.

Respect to another system, after droplets $(\sim 23 \mu \mathrm{L})$ were spontaneously pumped to the preset region on Track I (Fig. 5b (i)), two liquid bulges contacted each other and merged. Then the merged bulge touched three strips, connecting two preset regions (Track I) and the three tracks (Track II, 
Fig. $5 \mathrm{~b}$ (ii)). The pumping continued towards three preset regions on Track II until the end. During the pumping, the merged bulge de-bridged over two preset regions on Track I (Fig. 5b (iii)) first and then three strips on Track II (Fig. 5b (iv), see Supplementary Movie 7 for a complete cycle). This system integrates metering, merging, splitting and ultrafast long-range self-transport of droplets, which can be compounded in microfluidics to complete various complex tasks. To the best knowledge of the author, this functional system is proposed for the first time. Moreover, due to the scalability of the structure, the space between track I can be controlled by scaling down or enlarging the system in an equal proportion, to control the quantity of transported volume. Coupled with the advanced manufacturing technology, above two systems are even promising to realize the rational manipulation of droplets at micro-nano scale, which undoubtedly makes the proposed structure possess great application potential and market value in the future.

\section{Conclusions}

In summary, a multi-bioinspired surface which achieves the rational manipulation of droplets is proposed inspired by the Janus wettability of desert beetle, the tapered asymmetric of cacti spine, and the periodic characteristic structure of Nepenthes alata. Considerable droplets can be rapidly and remotely pumped from one site to another with ultralow loss. Additionally, the fantastic scalability of the structure enables it to rationally manipulate multi-scale (from micro/nano scale to millimeter scale) droplets. Two systems based on the bioinspired structure have been designed, which can be compounded in microfluidics to complete various complex tasks. This work provides new insights, from the design principle of multi-bioinspired surface to rational manipulation of droplets, reflecting its practical and potential applications from physics to biotechnology.

\section{Methods}

Chemicals. The following chemicals were obtained from Aladdin Industrial Corporation: $\mathrm{NaOH}$ ( $\geq 98 \%$ ), $\left(\mathrm{NH}_{4}\right)_{2} \mathrm{~S}_{2} \mathrm{O}_{8}(\geq 98 \%), \mathrm{C}_{6} \mathrm{H}_{14}(98 \%), \mathrm{C}_{18} \mathrm{H}_{37} \mathrm{Cl}_{3} \mathrm{Si}(>85 \%)$.

Sample fabrication. The silicon wafer was ultrasonically cleaned in acetone and isopropanol. Then we grew copper film of $\sim 600 \mathrm{~nm}$ on silicon wafer using magnetron sputtering at power of $300 \mathrm{~W}$ for 16 min. Sputtering was performed in Argon (99.99\% purity) under a working pressure of $0.5 \mathrm{~Pa}$. Superhydrophilic $\mathrm{Cu}(\mathrm{OH})_{2}$ nanowires were obtained by immersing the sample in the growth solution ( $0.625 \mathrm{M}$ sodium hydroxide and $0.025 \mathrm{M}$ ammonium persulfate aqueous solution) for 10 $\mathrm{min}$ at room temperature. The designed bio-inspired pattern involves the following steps. Positive photoresist (AZ4620) was spin-coated on $\mathrm{Cu}(\mathrm{OH})_{2}$ substrate with a photoresist spinner operating at $500 \mathrm{rpm}$ for $10 \mathrm{~s}$ and then at $1500 \mathrm{rpm}$ for $30 \mathrm{~s}$. Then the sample was placed on a hot plate at $95^{\circ} \mathrm{C}$ and baked for $10 \mathrm{~min}$. After that, the sample was developed by immersing in the AZ 400K developer for $100 \mathrm{~s}$ after exposure of UV light (Luminous intensity of $8 \mathrm{~mW} / \mathrm{cm}^{2}$ ) for $40 \mathrm{~s}$ with a mask aligner. Thereafter, the sample was immersing in n-hexane solution with octadecyltrichlorosilane (OTS, concentration of $5 \mathrm{mM}$ ) for $15 \mathrm{~min}$ to obtain the superhydrophobic surface. Finally, we performed lift-off by immersing the sample in the sodium hydroxide solution with a concentration of $3 \%$.

Finite Element Analysis. The CFD solver ANSYS FLUENT 19.0 is used for the 3D numerical solution of the discussed governing equations with the finite volume method. The problem is 
formulated as a transient laminar case with a second-order implicit temporal discretization, and a second order upwind spatial discretization. The VOF method is used to track the interface between the water and air phases. The Pressure Implicit with Splitting of Operators (PISO) algorithm was used for the velocity and pressure coupling. The time step was automatically adjusted with values in the range from $10^{-6}$ to $10^{-4} \mathrm{~s}$ in order to maintain the global Courant number below a value of 0.5 for calculation stability. Structured orthogonal meshes are adopted, and refined in the vertical direction of the droplet wetting wall boundary. The grid independence is verified based on the spreading distance of the droplet. After the current grid cell size is reduced to half, the change of the spreading distance of the droplet is less than one grid. In order to ensure the conservation of mass, the total volume of the liquid phase is monitored during the calculation process, and the volume change of the liquid phase is less than $1 \%$ when the calculation is stopped.

Determining droplets transport velocity. In these experiments, the volumes of droplet are 3,5, and $7 \mu \mathrm{L}$, respectively. The droplet dynamics were recorded by a high-speed camera at a frame rate of $1000 \mathrm{fps}$. The droplets transport velocities were obtained from the transport range $(L)$ and time $(t)$. A series of representative frames were taken from the high-speed video, and the time interval of each frame is $1 \mathrm{~ms}$. The moment that droplets touched the surface was defined as the starting point of the transport $(0 \mathrm{~ms})$. The moment that droplets arrived the preset site was recorded $(t \mathrm{~ms})$. Then the average velocity $(v)$ can be obtained as follows:

$$
v=\frac{L}{t}
$$

Noteworthily, the instantaneous velocities of droplets were represented by the displacement every $1 \mathrm{~ms}$. Each data represents readings averaged over at least five independent runs.

Characterization. The morphology of the Janus surface was characterized by scanning electron microscopy (SEM, Zeiss Ultra Plus) at an accelerating voltage of $5 \mathrm{kV}$. The composition of the nanowires was analyzed by energy dispersive spectroscopy (EDS). The water contact angle was measured with a DSA100 Drop Shape Analyzer system by depositing deionized water $(5 \mu \mathrm{L})$ on the surface at ambient temperature. The behaviors of droplets on the fabricated multi-bioinspired surface were recorded with a high-speed camera (VEO-710L, Phantom).

\section{Data availability}

All data needed to evaluate the conclusions in the paper are present in the paper and/or the Supplementary Information.

\section{References}

1. Stone, H. A., Stroock, A. D., Ajdari, A. Engineering flows in small devices: microfluidics toward a lab-on-a-chip. Annu. Rev. Fluid Mech. 36, 381-411 (2004).

2. Wang, H., Zhao, Z., Liu, Y., Shao, C., Bian, F., Zhao, Y. Biomimetic enzyme cascade reaction system in microfluidic electrospray microcapsules. Sci. Adv. 4, eaat2816 (2018).

3. Sun, Q., Wang, D., Li, Y., Zhang, J., Ye, S., Cui, J., et al. Surface charge printing for programmed droplet transport. Nat. Mater. 18, 936-941 (2019).

4. Damak, M., Varanasi, K. K. Electrostatically driven fog collection using space charge 
injection. Sci. $A d v$. 4, eaao5323(2018).

5. Park, K. C., Kim, P., Grinthal, A., He, N., Fox, D., Weaver, J. C., et al. Condensation on slippery asymmetric bumps. Nature 531, 78-82 (2016).

6. Li, C., Wu, L., Yu, C., Dong, Z., Jiang, L. Peristome-Mimetic Curved Surface for Spontaneous and Directional Separation of Micro Water-in-Oil Drops. Angew. Chem. Int. Ed. 129, 13811-13816 (2017).

7. Wang, Q., Yao, X., Liu, H., Quéré, D., Jiang, L. Self-removal of condensed water on the legs of water striders. Proc. Natl Acad. Sci. USA 112, 9247-9252 (2015).

8. Mouterde, T., Lehoucq, G., Xavier, S., Checco, A., Black, C. T., Rahman, A., et al. Antifogging abilities of model nanotextures. Nat. Mater. 16, 658-663 (2017).

9. Schutzius, T. M., Jung, S., Maitra, T., Graeber, G., Köhme, M., Poulikakos, D. Spontaneous droplet trampolining on rigid superhydrophobic surfaces. Nature 527, 82-85 (2015).

10. Wang, S., Lee, J. S., Wahiduzzaman, M., Park, J., Muschi, M., Martineau-Corcos, C., et al. A robust large-pore zirconium carboxylate metal-organic framework for energy-efficient water-sorption-driven refrigeration. Nat. Energy 3, 985-993 (2018).

11. Amini, S., Kolle, S., Petrone, L., Ahanotu, O., Sunny, S., Sutanto, C. N., et al. Preventing mussel adhesion using lubricant-infused materials. Science 357, 668-673 (2017).

12. Lv, J. A., Liu, Y., Wei, J., Chen, E., Qin, L., Yu, Y. Photocontrol of fluid slugs in liquid crystal polymer microactuators. Nature 537, 179-184 (2016).

13. Liu, M., Wang, S., Jiang, L. Nature-inspired superwettability systems. Nat. Rev. Mater. 2, $1-17$ (2017).

14. Parker, A. R., Lawrence, C. R. Water capture by a desert beetle. Nature 414, 33-34 (2001).

15. Ju, J., Bai, H., Zheng, Y., Zhao, T., Fang, R., Jiang, L. A multi-structural and multifunctional integrated fog collection system in cactus. Nat. Commun. 3, 1-6 (2012).

16. Chen, H., Zhang, P., Zhang, L., Liu, H., Jiang, Y., Zhang, D., et al. Continuous directional water transport on the peristome surface of Nepenthes alata. Nature 532, 85-89 (2016).

17. Bentley, P. J., Blumer, W. F. C. Uptake of water by the lizard, Moloch horridus. Nature 194, 699-700 (1962).

18. Zheng, Y., Bai, H., Huang, Z., Tian, X., Nie, F. Q., Zhao, Y., et al. Directional water collection on wetted spider silk. Nature 463, 640-643 (2010).

19. Wang, T., Si, Y., Dai, H., Li, C., Gao, C., Dong, Z., et al. Apex structures enhance water drainage on leaves. Proc. Natl Acad. Sci. USA 117, 1890-1894 (2020).

20. Prakash, M., Quéré, D., Bush, J. W. Surface tension transport of prey by feeding shorebirds: the capillary ratchet. Science 320, 931-934 (2008).

21. Comanns, P., Buchberger, G., Buchsbaum, A., Baumgartner, R., Kogler, A., Bauer, S., et al. Directional, passive liquid transport: the Texas horned lizard as a model for a biomimetic 'liquid diode'. J. R. Soc. Interface 12, 20150415 (2015).

22. Daniel, S., Chaudhury, M. K., Chen, J. C. Fast drop movements resulting from the phase change on a gradient surface. Science 291, 633-636 (2001). 
23. Li, J., Zhou, X., Li, J., Che, L., Yao, J., McHale, G., et al. Topological liquid diode. Sci. Adv. 3, eaao3530 (2017).

24. Chu, K. H., Xiao, R., Wang, E. N. Uni-directional liquid spreading on asymmetric nanostructured surfaces. Nat. Mater. 9, 413-417 (2010).

25. Bai, H., Wang, L., Ju, J., Sun, R., Zheng, Y., Jiang, L. Efficient water collection on integrative bioinspired surfaces with star-shaped wettability patterns. Adv. Mater. 26, 50255030 (2014).

26. Li, K., Ju, J., Xue, Z., Ma, J., Feng, L., Gao, S., et al. Structured cone arrays for continuous and effective collection of micron-sized oil droplets from water. Nat. Commun. 4, 1-7 (2013).

27. Jiang, J., Gao, J., Zhang, H., He, W., Zhang, J., Daniel, D., et al. Directional pumping of water and oil microdroplets on slippery surface. Proc. Natl Acad. Sci. USA 116, 2482-2487 (2019).

28. Zhao, Z., Li, C., Dong, Z., Yang, Y., Zhang, L., Zhuo, S., et al. Adaptive superamphiphilic organohydrogels with reconfigurable surface topography for programming unidirectional liquid transport. Adv. Funct. Mater. 29, 1807858 (2019).

29. Li, C., Yu, C., Zhou, S., Dong, Z., Jiang, L. Liquid harvesting and transport on multiscaled curvatures. Proc. Natl Acad. Sci. USA 117, 23436-23442 (2020).

30. Vialetto, J., Hayakawa, M., Kavokine, N., Takinoue, M., Varanakkottu, S. N., Rudiuk, S., et al. Magnetic actuation of drops and liquid marbles using a deformable paramagnetic liquid substrate. Angew. Chem. Int. Ed. 56, 16565-16570 (2017).

31. Bjelobrk, N., Girard, H. L., Subramanyam, S. B., Kwon, H. M., Quéré, D., Varanasi, K. K. Thermocapillary motion on lubricant-impregnated surfaces. Phys. Rev. Fluids 1, 063902 (2016).

32. Ichimura, K., Oh, S. K., Nakagawa, M. Light-driven motion of liquids on a photoresponsive surface. Science 288, 1624-1626 (2000).

33. Zang, D., Yi, H., Gu, Z., Chen, L., Han, D., Guo, X., et al. Interfacial engineering of hierarchically porous $\mathrm{NiTi} /$ hydrogels nanocomposites with exceptional antibiofouling surfaces. Adv. Mater. 29, 1602869 (2017).

34. Li, C., Li, N., Zhang, X., Dong, Z., Chen, H., Jiang, L. Uni-directional transportation on peristome-mimetic surfaces for completely wetting liquids. Angew. Chem. Int. Ed. 55, 14988-14992 (2016).

35. Cheng, Z., Zhang, D., Luo, X., Lai, H., Liu, Y., Jiang, L. Superwetting shape memory microstructure: Smart wetting control and practical application. Adv. Mater. 33, 2001718 (2021).

36. Wu, D., Zhang, Z., Zhang, Y., Jiao, Y., Jiang, S., Wu, H., et al. High-Performance Unidirectional Manipulation of Microdroplets by Horizontal Vibration on Femtosecond Laser-Induced Slant Microwall Arrays. Adv. Mater. 32, 2005039 (2020).

37. Ge, P., Wang, S., Zhang, J., Yang, B. Micro-/nanostructures meet anisotropic wetting: from 
preparation methods to applications. Mater. Horiz. 7, 2566-2595 (2020).

38. Geng, H., Bai, H., Fan, Y., Wang, S., Ba, T., Yu, C., et al. Unidirectional water delivery on a superhydrophilic surface with two-dimensional asymmetrical wettability barriers. Mater. Horiz. 5, 303-308 (2018).

39. Long, D., Yang, W., Scanlon, B. R., Zhao, J., Liu, D., Burek, P., et al. South-to-North Water Diversion stabilizing Beijing's groundwater levels. Nat. Commun. 11, 1-10 (2020).

40. Quéré, D. Wetting and roughness. Annu. Rev. Mater. Res.38, 71-99 (2008).

41. Ma, H., Cao, M., Zhang, C., Bei, Z., Li, K., Yu, C.,et al. Directional and Continuous Transport of Gas Bubbles on Superaerophilic Geometry-Gradient Surfaces in Aqueous Environments. Adv. Funct. Mater. 28, 1705091 (2018).

42. Gau, H., Herminghaus, S., Lenz, P., Lipowsky, R. Liquid morphologies on structured surfaces: from microchannels to microchips. Science 283, 46-49 (1999).

43. Hirai, Y., Mayama, H., Matsuo, Y., Shimomura, M. Uphill water transport on a wettabilitypatterned surface: experimental and theoretical results. ACS Appl. Mater. Inter. 9, 1581415821 (2017).

44. Alheshibri, M. H., Rogers, N. G., Sommers, A. D., Eid, K. F. Spontaneous movement of water droplets on patterned $\mathrm{Cu}$ and $\mathrm{Al}$ surfaces with wedge-shaped gradients. Appl. Phys. Lett. 102, 174103 (2013)

45. Ghosh, A., Ganguly, R., Schutzius, T. M., Megaridis, C. M. Wettability patterning for highrate, pumpless fluid transport on open, non-planar microfluidic platforms. Lab Chip 14, 1538-1550 (2014).

46. Chen, H., Ran, T., Gan, Y., Zhou, J., Zhang, Y., Zhang, L.,et al. Ultrafast water harvesting and transport in hierarchical microchannels. Nat. Mater. 17, 935-942 (2018).

47. Schutzius, T. M., Elsharkawy, M., Tiwari, M. K., Megaridis, C. M. Surface tension confined (STC) tracks for capillary-driven transport of low surface tension liquids. Lab Chip $\mathbf{1 2}$, 5237-5242 (2012).

\section{Competing interests}

The authors declare no competing financial interests. 
Figures and Tables

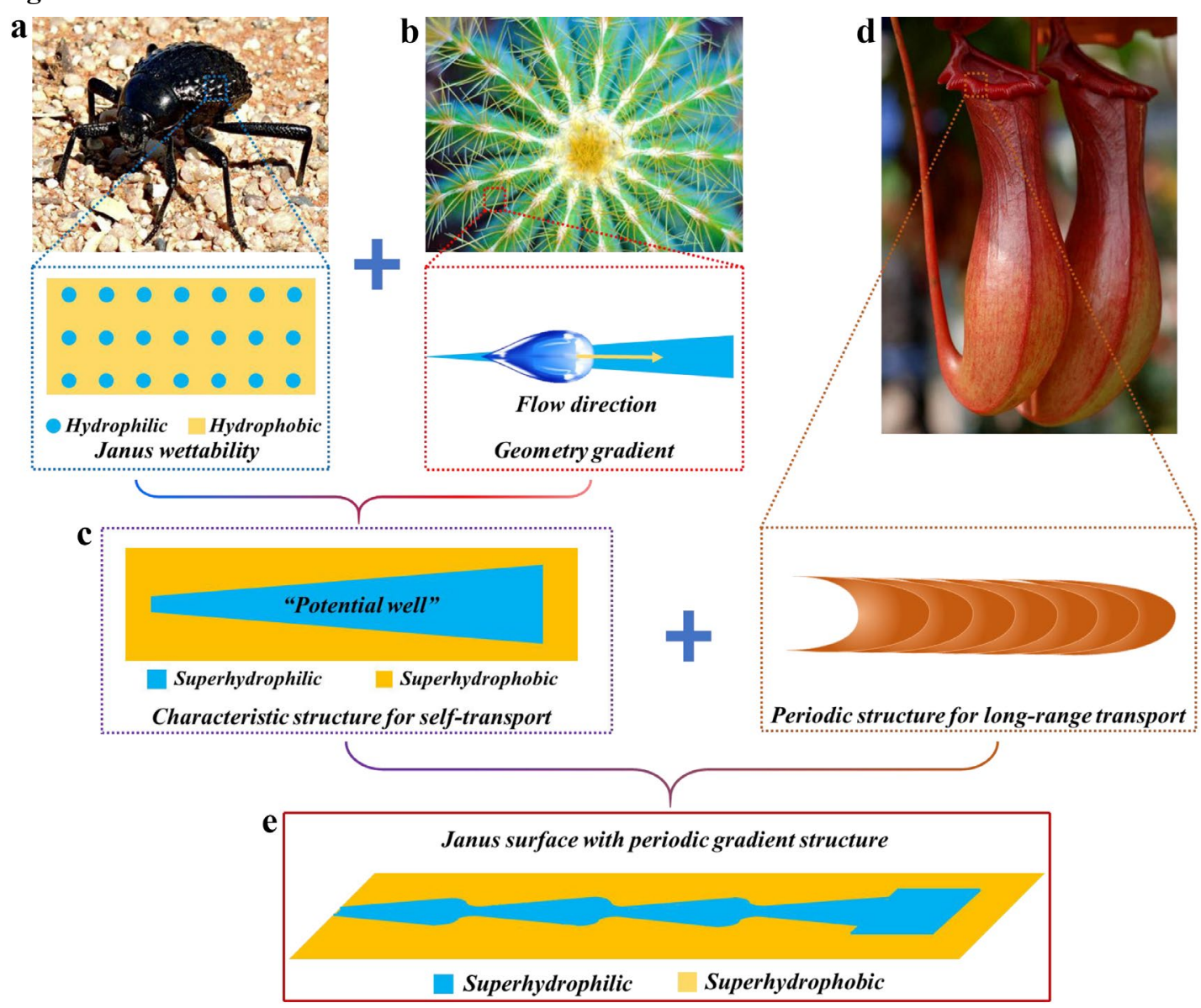

Fig. 1 Overview of the design principle of the multi-bioinspired surface with 2D periodic gradient structures. a The Janus wettability derived from desert beetle. $\mathbf{b}$ The anisotropic shape of cactus spines possesses geometric gradient, which contributes to self-propelled droplet transport. c The periodic characteristic structures (microcavities) of Nepenthes alata are responsible for continuous long-range transport of droplets. d Characteristic structure integrating the Janus wettability and geometry gradient. e The designed Janus surface comprising periodic asymmetric structures. 

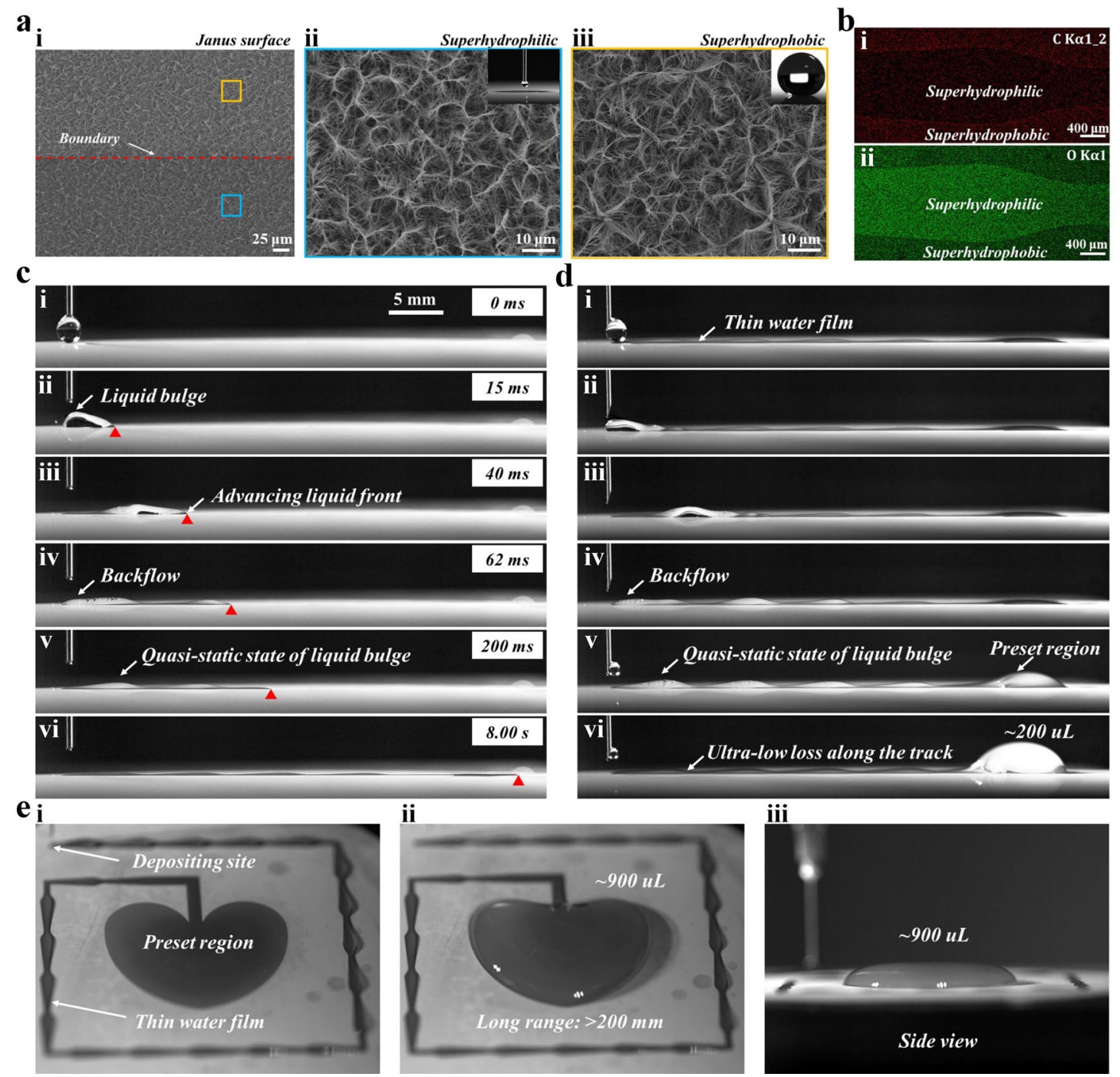

$62 m s$
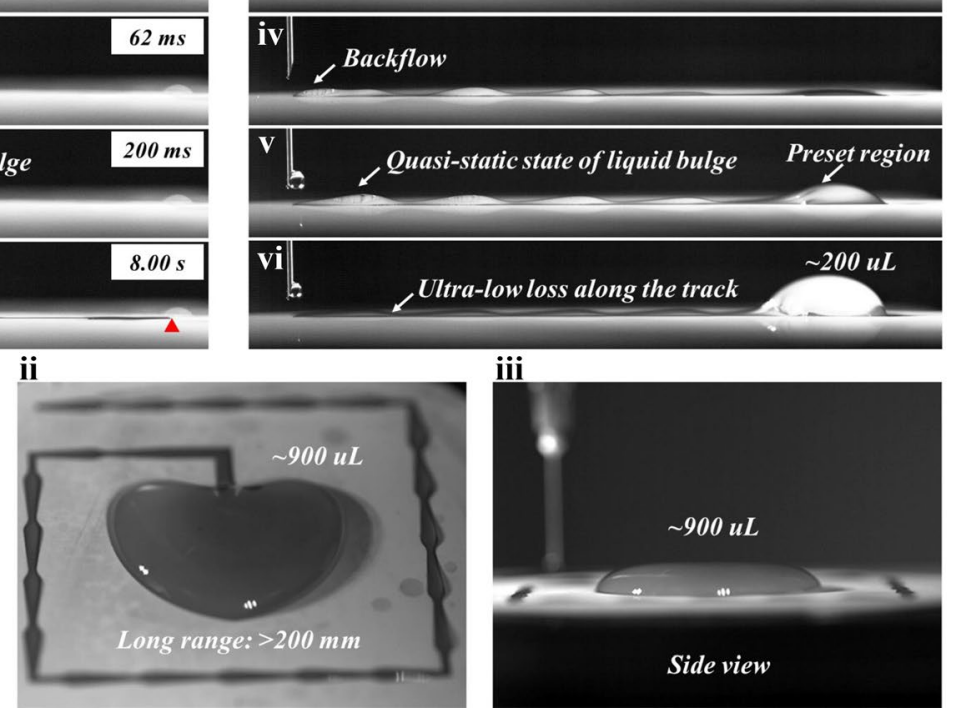

Fig. 2 The fabricated multi-bioinspired surface with 2D periodic structures, achieving the ultrafast and long-range self-transport of droplets. a Scanning electron microscopy image of (i) the fabricated Janus surface, (ii) superhydrophilic area and (iii) superhydrophobic area, where nanowires of $\mathrm{Cu}(\mathrm{OH})_{2}$ can be observed. The insets show the water contact angle at corresponding area. b Energy dispersive spectroscopy results of the Janus surface. $\mathbf{c}$ Time-lapse images of droplet $(7 \mu \mathrm{L})$ transport behaviors on the dry superhydrophilic track. d Time-lapse images of transport behaviors of considerable droplets $(\sim 200 \mu \mathrm{L})$ on the wet superhydrophilic track. e Time-lapse images illustrating the advantages of proposed structure in long-range $(>200 \mathrm{~mm})$ self-transport of numerous droplets $(\sim 900 \mu \mathrm{L})$. 
a

i
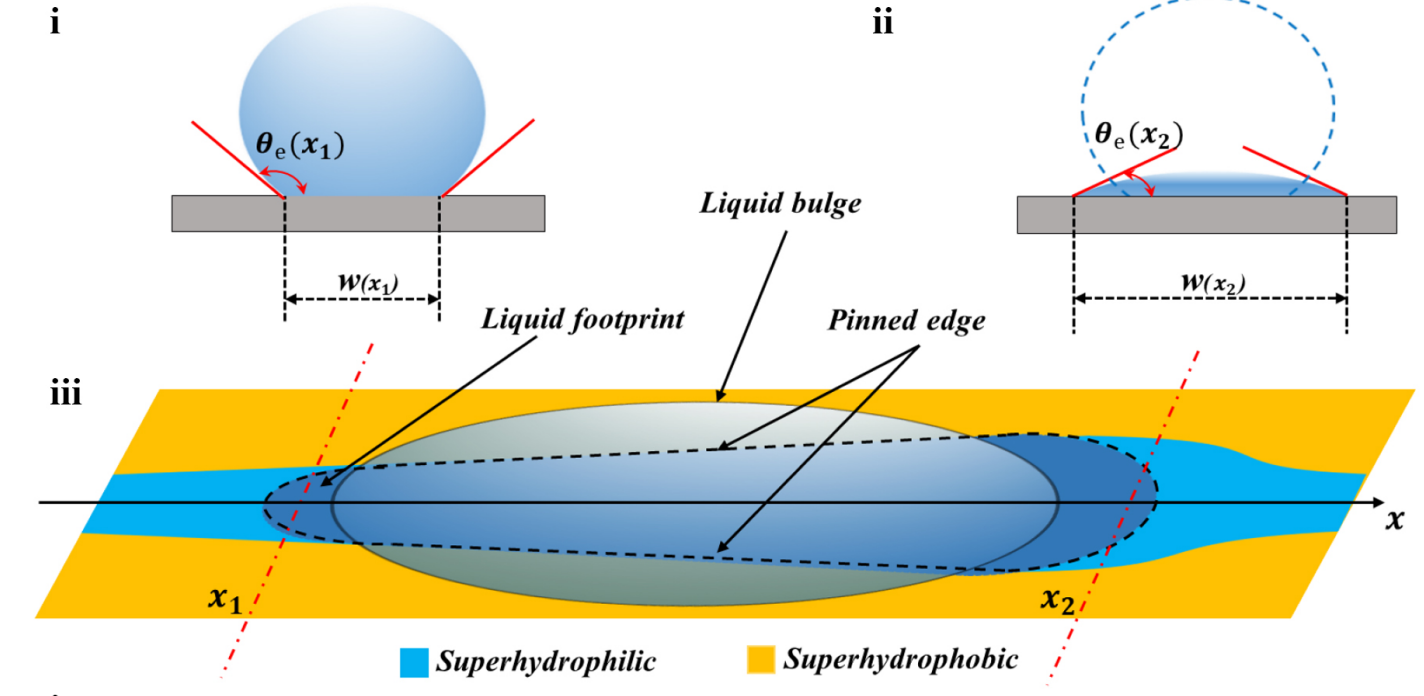

Liquid bulge

iv

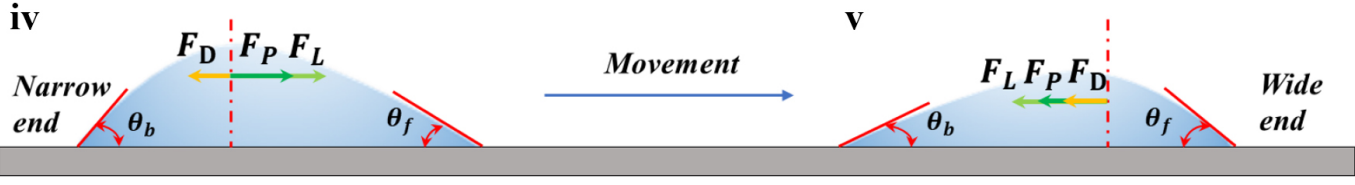

b

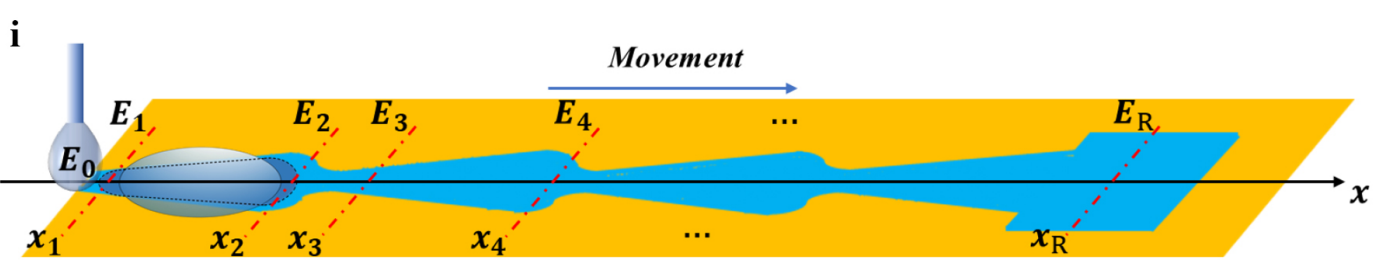

For droplets on the dry track, $E_{1}>E_{2}>E_{3}>E_{4}>\cdots>E_{R}$

ii

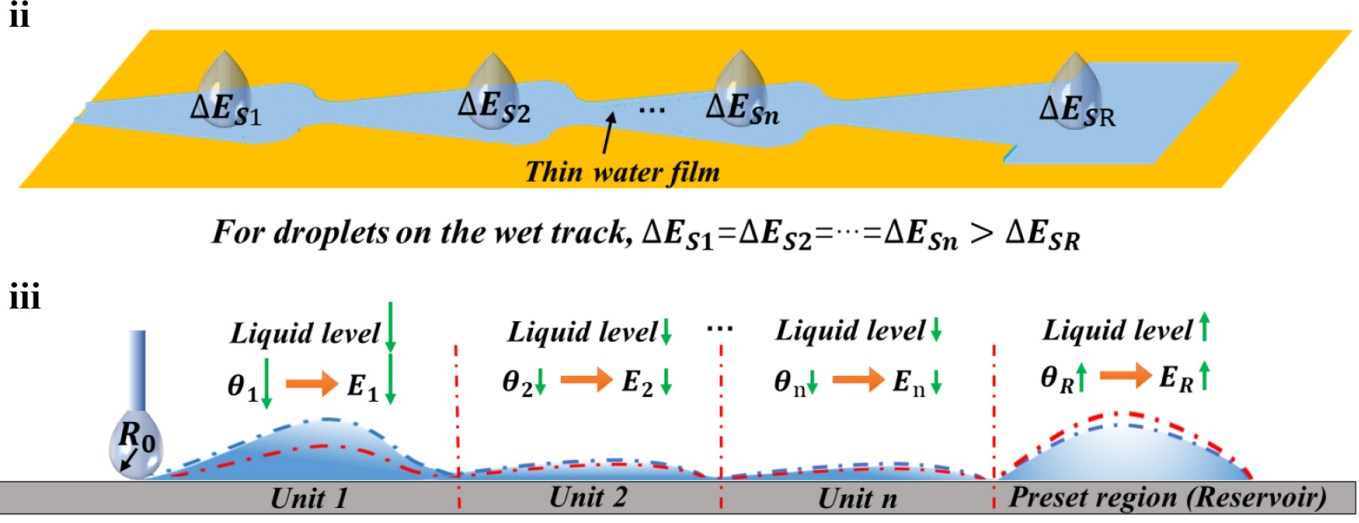

In quasi-static state, $\Delta E_{1}+\Delta E_{2}+\cdots+\Delta E_{\mathrm{n}}>\Delta E_{R}$

Fig. 3 Schematic analysis of the droplet transport process on fabricated multi-bioinspired surface. a Schematic image illustrating origin of the force on the liquid bulge. (i-iii) A droplet placed on the Janus surface of a unit structure having confinement width $w$, and $\theta_{\mathrm{e}}$ varies along the transport length $x$. They are three dominant forces acting on the liquid bulge, namely the Laplace force $\left(F_{\mathrm{L}}\right)$ arising from the shape gradient, the pinning force $\left(F_{\mathrm{P}}\right)$ derived from the Janus structure, and the hydrodynamic resistance force $\left(F_{\mathrm{D}}\right)$ formed by the surface of the track. (iv) When the droplet is at the narrow end of a unit, $F_{\mathrm{L}}$ and $F_{\mathrm{P}}$ are the driving force and $F_{\mathrm{D}}$ is the resistant force. (v) When the droplet is at the far downstream portion of a unit, $F_{\mathrm{L}}, F_{\mathrm{P}}$, and $F_{\mathrm{D}}$ are the resistant force. b Schematic images illustrating the underlying mechanism from the perspective of system energy. 
(i) Droplet spread over the entire dry superhydrophilic track, as that leads to declining of $\theta_{i}$, resulting in a lower surface energy. (ii) The increased surface energy $\left(\Delta E_{S i}, i=1-n\right)$ of each unit due to the introduction of a droplet $\left(V_{0}\right)$ is theoretically equal due to the periodicity, which is higher than that of the preset region owing to the confinement effect. (iii) The reduced surface energy $\left(\Delta E_{i}, i=1\right.$ n) of the superhydrophilic track is larger than that increased of the preset region. 

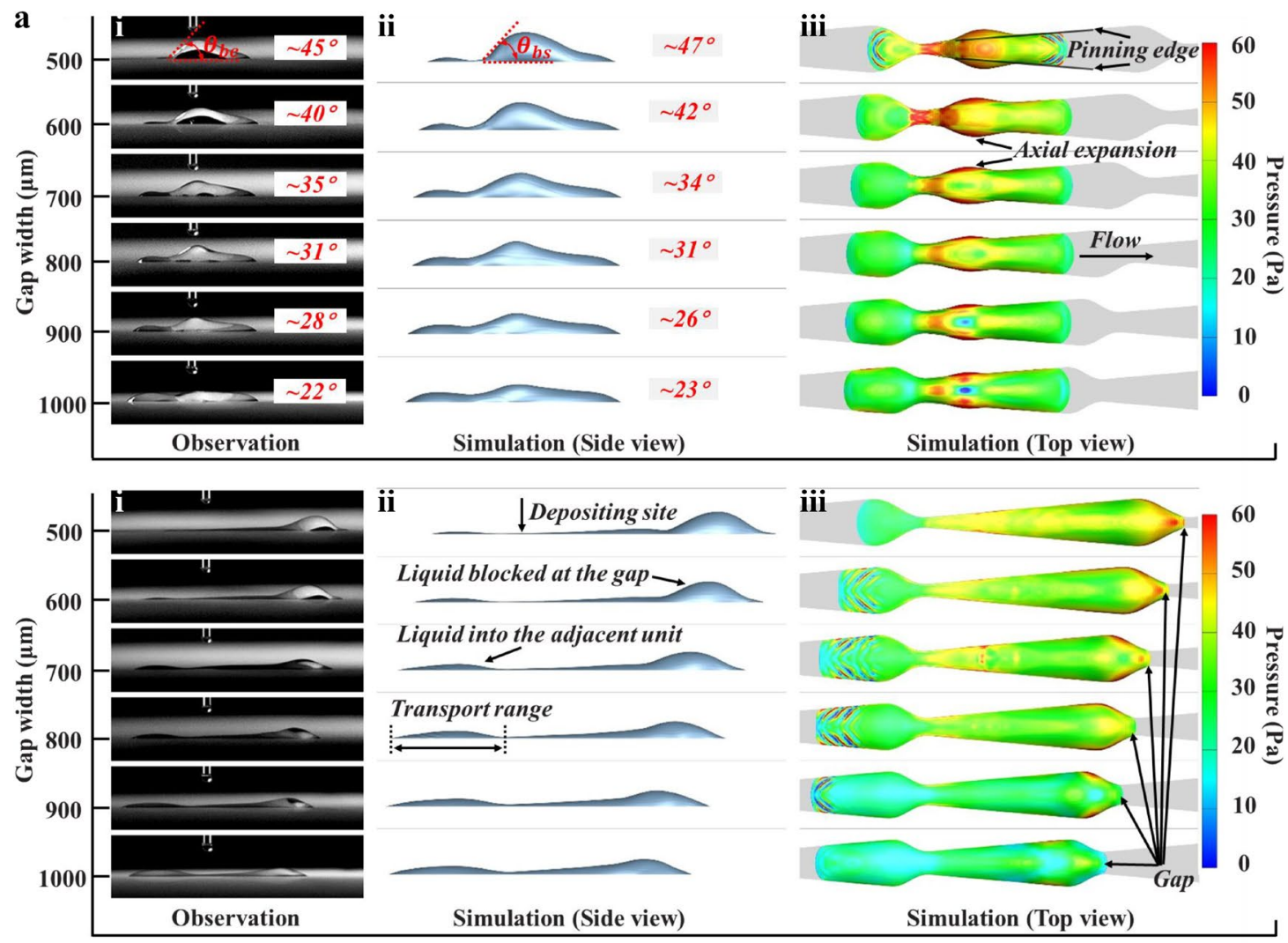

c

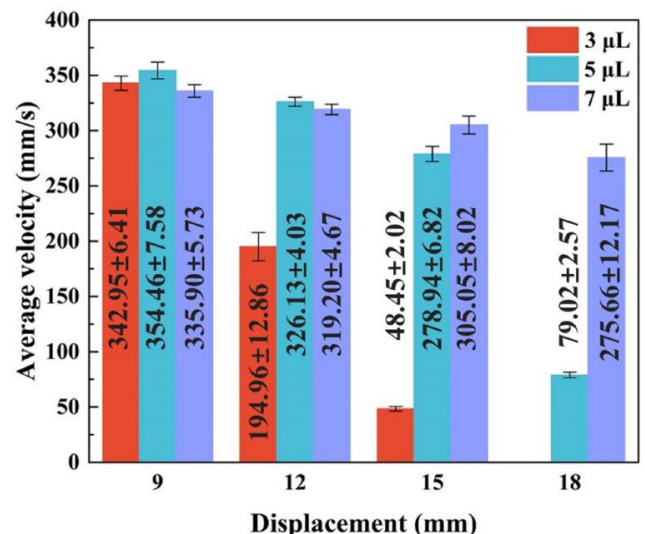

e

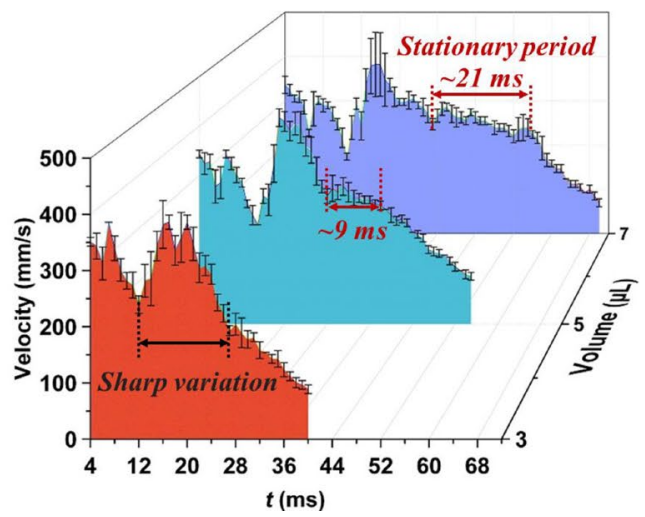

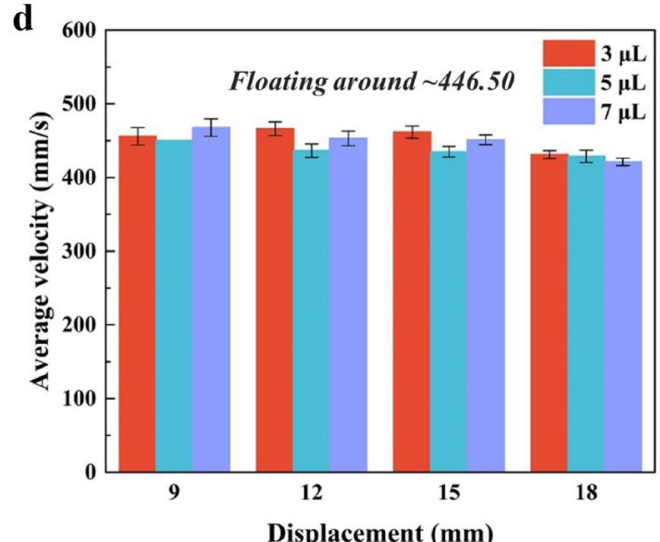

f

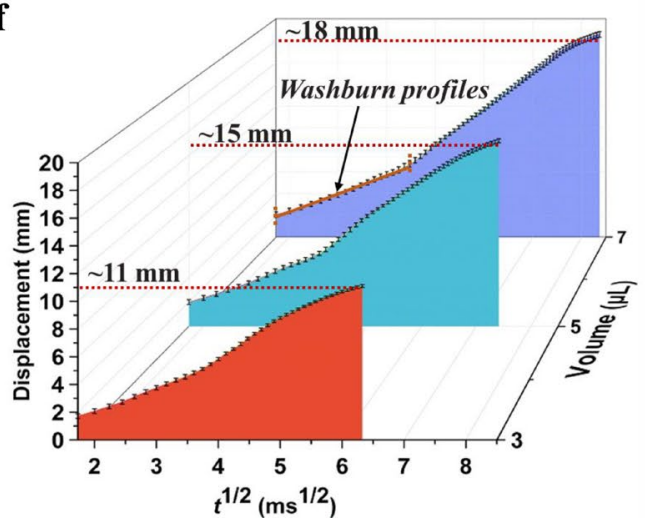

Fig. 4 Droplets transport performance and dynamic characteristics on the fabricated multibioinspired surface. a,b Effect of the critical parameter of the novel structure namely gap width on the droplet transport in the early stage (a, divorced from the syringe needle) and the final stage ( $\mathbf{b}$, transported to the end of the current unit). The gap widths are 500,600, 700, 800, 900 and $1000 \mu \mathrm{m}$, respectively. 
$\theta_{b e}$ and $\theta_{b s}$ denote the backward contact angle in experimental observation and simulation result. c,d Average velocity of droplet transport on the dry (c) or wet (d) superhydrophilic track with different volume $(3,5$, and $7 \mu \mathrm{L})$ in different displacement $(\sim 9, \sim 12, \sim 15$, and $\sim 18 \mathrm{~mm})$. e Instantaneous velocity $(\Delta \mathrm{t}=1 \mathrm{~ms})$ of droplet as a function of transport time with different volume $(3,5$, and $7 \mu \mathrm{L})$. f Distance from the depositing site as a function of $t^{1 / 2}$. 
$\mathbf{a}$

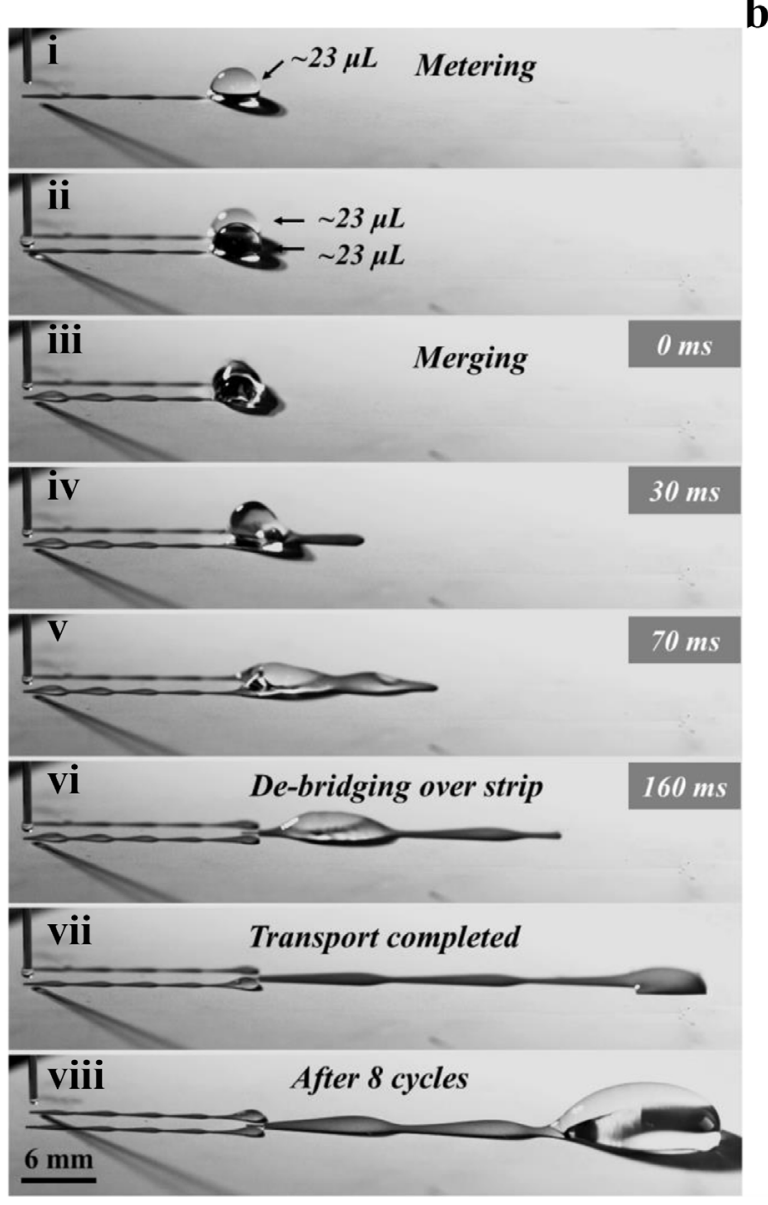

b

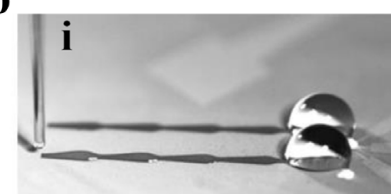

\section{Metering}
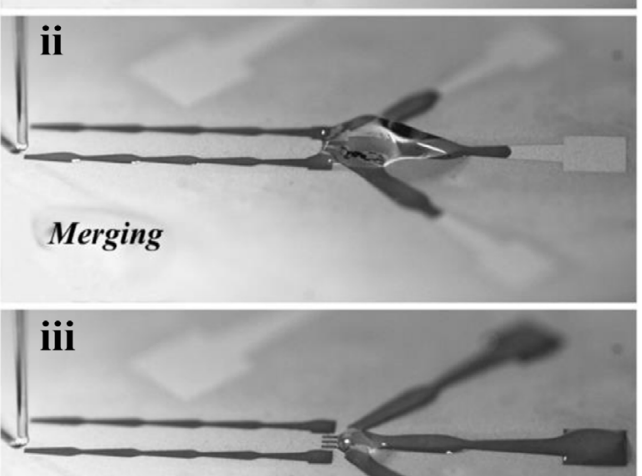

De-bridging over strip

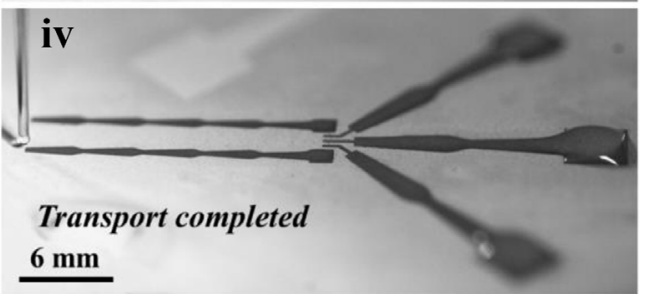

c
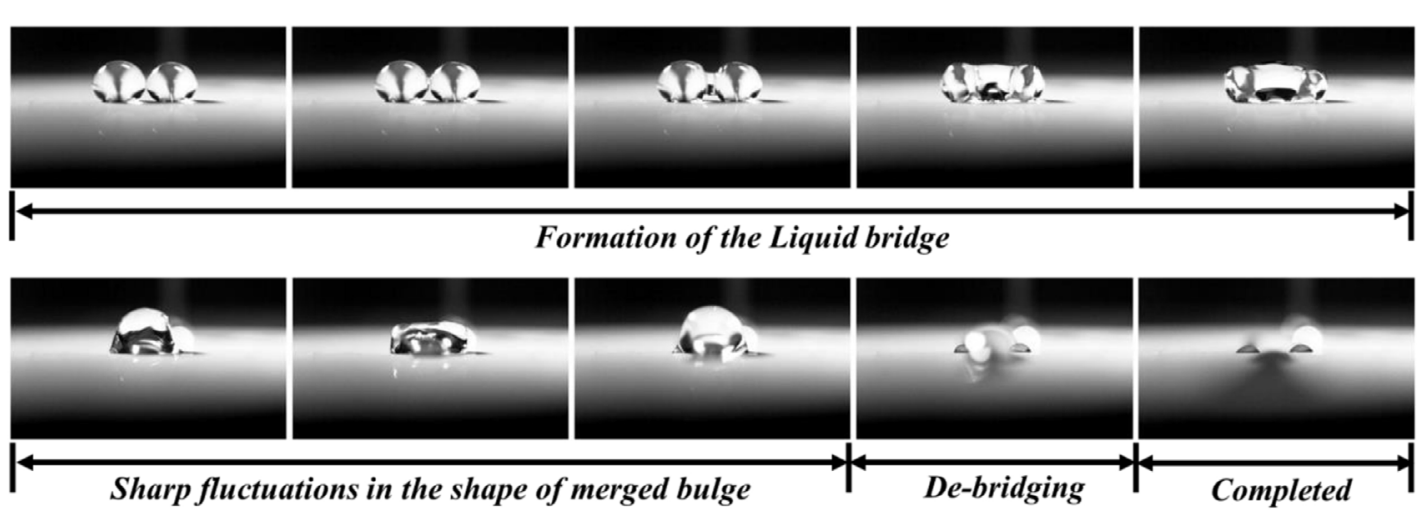

Fig. 5 Two systems based on the bioinspired structure to complete complex tasks on open surface. a A system that integrates metering (i-ii), merging (iii) and ultrafast long-range selftransport of droplets (iv-vii). The process is highly repeatable (after 8 cycles, viii). b A system that integrates metering (i), merging (ii), splitting (iii) and ultrafast long-range self-transport of droplets (iv). c Snapshots of the formation of liquid bridge, sharp fluctuations in the shape of merged bulge, de-bridging over strip of merged bulge and final state of the liquid bulges at the preset region on Track I (Side view). 


\section{Supplementary Files}

This is a list of supplementary files associated with this preprint. Click to download.

- ElectronicSupplementaryMaterial.docx

- SupplementaryMovie1.mov

- SupplementaryMovie2.mov

- SupplementaryMovie3.mov

- SupplementaryMovie4.mov

- SupplementaryMovie5.mov

- SupplementaryMovie6.mov

- SupplementaryMovie7.mov 